\title{
Origami-Induced Alignment of MNP Inside of DNA Nanotubes
}

\author{
Adele Rafati, ${ }^{1,2^{*}}$, Ali Zarrabi ${ }^{3}$ and Pooria Gill ${ }^{1,4}$ \\ ${ }^{1}$ Department of Medical Nanotechnology, Faculty of Advanced Technologies in Medicine, Mazandaran \\ University of Medical Sciences, Sari, Iran \\ ${ }^{2}$ Diabetes Research Center, Mazandaran University of Medical Sciences, Sari, Iran \\ ${ }^{3}$ Nanotechnology Research and Application Center, Sabanci University, Sabanci, Turkey \\ ${ }^{4}$ Nanomedicine Group, Immunogenetics Research Center, Mazandaran University of Medical Sciences, \\ Sari, Iran
}

*Corresponding author: Adele Rafati, Department of Medical Nanotechnology, Faculty of Advanced Technologies in Medicine, Mazandaran University of Medical Sciences, Sari, Iran

\begin{abstract}
Background: The advent of nanotubes in the nanotechnology world has led to significant advances in a number of biological and materials application, due to their structural properties such as the surface to volume ratio and potential to surface carrying or inside capsulation any materials. Among the various types of nanotubes, DNA nanotubes, due to their unique characteristics, such as precis controllability and programmability in shape/size/length/diameter and its biological origin compared to different types of nanomaterial, can be the suitable candidate for template patterning alignment and precise organization of nanoparticles at surface or into channel. These characteristics can be used in nanoelectronic devise or in the field of diagnostic nanobiosensores.
\end{abstract}

Methods: Here, we report a new construction methodology for encapsulation of magnetic nanoparticles inside DNA nanotubes channel. Constructed-simultaneously encapsulation of magnetic nanoparticles into the large channel of this tubes leads to "pea-pod" particle alignment in nanotube channel.

Results: Transmission electron microscopy and atomic force microscopy confirmed the fabrication of DNA nanotubes contained the magnetic nanoparticles inside the channel.

Conclusion: These biohybrid nanomaterial would be proposed as the nanoarray platform in nanobiosensing devices.

\section{Keywords}

DNA nanotubes, Magnetic nanoparticles, Transmission electron microscopy, Atomic force microscopy, Nanobiosensors

\section{Introduction}

Deoxyribonucleic acid (DNA) has recently suggested opportunities for the fabrications of nanostructures and massively construction of the artificial nanoarchitectures with various geometries or patterns by the self-assembly phenomena [1-6]. Scaffolded DNA origami has become a unique technique for designing well-defined nanostructures with any desired shape and pattern because of programmability and controllability in fabrication and for the controlled arrangement of functional nanostructures with few nanometer resolutions [7,8]. DNA nanotechnology also has used DNA strands to assemble gold, Magnetic and other nanoparticles into distinct 1, 2-D structures, or into periodic $3-D$ crystals $[9,10]$. Combining the novel electronic, magnetic, and optical properties of either semiconductor or metallic nanoparticles with the self-assembly properties of DNA strands can led to construction of new hybrid bio-nanomaterial with ability to precise organization and alignment of nanoparticles [11,12]. These unique features make DNA origami nanostructures appropriate candidates for use as platform to alignment of nanoparticles for nanodevices fabrication. Consequently, a number of studies have shown the precise organization of metallic nanoparticles on/ in various DNA origami shapes $[13,14]$.

Among the various types of DNA nanostructures, DNA nanotubes, due to their unique characteristics, such as surface-to-volume ratio, controllability and pro-

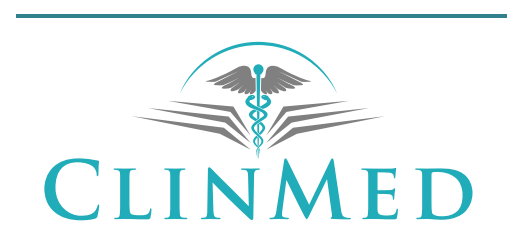

INTERNATIONAL LIBRARY
Citation: Rafati A, Zarrabi A, Gill P (2020) Origami-Induced Alignment of MNP Inside of DNA Nanotubes. Int J Med Nano Res 7:031. doi.org/10.23937/2378-3664/1410031

Accepted: February 18, 2020: Published: February 20, 2020

Copyright: (c) 2020 Rafati A, et al. This is an open-access article distributed under the terms of the Creative Commons Attribution License, which permits unrestricted use, distribution, and reproduction in any medium, provided the original author and source are credited. 
grammability in size and fabrication, ability to carry material over the surface, encapsulation of material inside the channel and its biological origin compared to different types of nanomaterial, has a vast potential for application such as patterning to precise organization of nanoparticles that can be used as a sensing platform in the field of diagnostic nanobiosensors [1517]. In fact, DNA nanotubes are filamentous structures that are made from double-stranded DNA helix by bottom-up or top-down techniques with a hollow central channel, and walls made of DNA [18].

The most important applications of DNA nanostructures coupled with nanoparticles are their ability to arrange some materials on them, which directly relate to the programmability of DNA molecules. These nanostructures could serve as the excellent platforms for carrying nanoparticles, antibodies, and protein while the functional groups were incorporated inside and outside of the nanomaterials [19-23]. Periodic array of nanoparticles on surface or in channel of DNA nanotubes explored their potentials for the several applications such as sensing platform in nanobiosensors or DNA nanowire fabrication [15-17]. The fabrications of DNA nanotubes particularly with the incorporations of 1-D nanoparticles such as metallic and magnetic nanoparticles have expanded their applications in nanotheraputics and nanodiagnostics $[5,6,19,20]$. Physical properties of the nanocomplexes change with the incorporation of the nanoparticles within the DNA nanotubes $[21,22]$.

The improvement of any aspect of these physical characteristics could greatly expand the range of employing theses nanoarchitectures in various areas of the science and technology [15-23].

DNA nanotubes could be incorporated with the nanoparticles through the non-covalently binding to the positively charged nanoparticles; however, for achieving the surface patterning or positioning of DNA nanotubes by nanoparticles could also being possible by covalently attaching the functional groups or molecules [24,25].

In this study we described a new procedure for the origami induced alignment of cationic-charged magnetic nanoparticles inside DNA nanotubes (Figure 1). The incorporation of the positively charged nanoparticles in the DNA nanotubes can be occurred simultaneously within a DNA-origami self-assembly reaction. By this methodology, DNA nanotubes can be suggested as nanocarriers; however, some novel physicochemical properties would be investigated from the nanotubes due to their carrying nanoparticles.

Theses nanotubes were characterized by transmission electron and atomic force microscopes. Also, their electrophoretic nobilities were studied using the gel electrophoresis experiment.

\section{Methods}

\section{Computational modelling of DNA nanotube}

The cadnano software was used for designing desired DNA nanotubes in this study. DNA sequence of M13mp18 phage genome was applied in the software as the scaffolded strand to achievement of oligonucleotides sequences of DNA staple as a helper for self-assembly of scaffolded strand into DNA nanotubes. The staple strands sequence was determined based on their complementary with the special site of scaffold that bind to form desired structure. The honeycomb style was introduced in the cadnano for properly folding of DNA nanotubes within a DNA origami reaction.

\section{Fabrication of DNA nanotubes via self-assembly DNA origami}

The origami reaction was prepared by mixing $20 \mathrm{nM}$ DNA scaffold M13mp18 single-stranded DNA (from New England Biolabs; Massachusetts, USA) with $100 \mathrm{nM}$ of each staple oligonucleotide (synthesized and desalted by Bioneer; Korea) that those were prepared in $1 \mathrm{X}$ Tris, acetic acid, and EDTA buffer $(40 \mathrm{mM}$ Tris-acetic acid buffer, $\mathrm{pH}$ 8.0, and $12.5 \mathrm{mM}$ magnesium acetate). The mixture was kept at $95^{\circ} \mathrm{C}$ for 5 min for denaturation of undesired secondary structures of any strand and between them and then cooled from $95{ }^{\circ} \mathrm{C}$ to 20 ${ }^{\circ} \mathrm{C}$ with a constant rate of $-1{ }^{\circ} \mathrm{C} / \mathrm{min}$ in the Rotor Gene Q thermocycler (QIAGEN, Germany) for annealing of desired shape. For the fabrication of long and sturdier DNA nanotubes, the origami products were treated by ligation process with T4 DNA ligase. The ligation-reaction mix was prepared containing $2 \mu \mathrm{L}$ of 10X T4 DNA

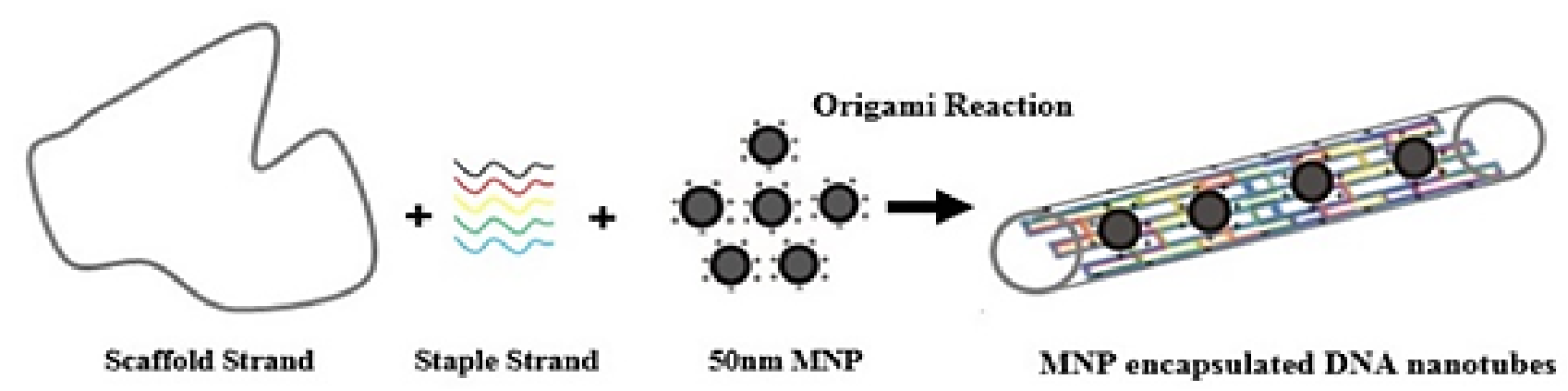

Figure 1: Schematic graphical view of DNA nanotube filled up with the magnetic nanoparticles. 
ligase reaction buffer, $10 \mu \mathrm{L}$ of self-assembled DNA nanotubes, $2 \mu \mathrm{L}$ of $50 \%$ polyethylene glycol, and $1 \mu \mathrm{L}$ of T4 DNA ligase enzyme. Finally, the ligation mix was incubated at $37^{\circ} \mathrm{C}$ for 1 hour.

\section{Encapsulation and alignment of MNP inside DNA nanotube channel}

For fabrication of DNA nanotubes including magnetic nanoparticles inside the channel, cationic-charged MNPs (fluidMAG-UC/C: Cationic charged, with $50 \mathrm{~nm}$ hydrodynamic dimension measured by the manufacturer (Chemicell GmbH, Germany) with dynamic light scattering; DLS) were used with the hypothesis that these MNPs have tendency to physical adsorption to DNA strands and was stabilized by cross-linking with the internal negative charge of DNA nanotube resulting from anionic phosphate group. By considering it, fabrication of DNA nanotube by top-down method was accompanied and joined by including process of cationic charged MNP (C-MNP). For this purpose, 2.5 $\times 10^{-3} \mathrm{mg} / \mathrm{ml} \mathrm{C-MNP}$ (from Chemicell GmbH, Germany) was combined with the Origami reaction mix of DNA nanotube. The origami reaction was prepared by mixing 20 nM DNA scaffold M13mp18 single-stranded DNA (from New England Biolabs; Massachusetts, USA) with $100 \mathrm{nM}$ of each staple oligonucleotide (synthesized and desalted by Bioneer; Korea) that those were prepared in $1 \mathrm{X}$ Tris, acetic acid, and EDTA buffer (40 $\mathrm{mM}$ Tris-acetic acid buffer, $\mathrm{pH} 8.0$, and $12.5 \mathrm{mM}$ magnesium acetate). The mixture was kept at $95{ }^{\circ} \mathrm{C}$ for 5 min and then annealed from $95{ }^{\circ} \mathrm{C}$ to $20^{\circ} \mathrm{C}$ with a constant rate of $-1{ }^{\circ} \mathrm{C} / \mathrm{min}$ in the Rotor Gene $\mathrm{Q}$ thermocycler (QIAGEN, Germany). For the fabrication of long and sturdier DNA nanotubes, the origami products were treated by ligation process with T4 DNA ligase. The ligation-reaction mix was prepared containing $2 \mu \mathrm{L}$ of 10X T4 DNA ligase reaction buffer, $10 \mu \mathrm{L}$ of self-assembled DNA nanotubes, $2 \mu \mathrm{L}$ of $50 \%$ polyethylene glycol, and $1 \mu \mathrm{L}$ of T4 DNA ligase enzyme. Finally, the ligation mix was incubated at $37{ }^{\circ} \mathrm{C}$ for 1 hour. Then, the fabricated magnetic DNA nanotubes were separated from simple DNA nanotubes and others by Magneto PURE-Micro separator (from Chemicell GmbH, Germany).

\section{Gel electrophoresis of DNA nanotubes encapsu- lated with C-MNPs}

For assessing the electrophoretic behaviors of these nanotubes filled-up with magnetic nanoparticles, $5 \mu \mathrm{L}$ of the nanotubes were loaded in $1 \%$ agarose gel and the assay was run within an electrophoresis experiment at 0.5X TBE buffer $\mathrm{pH}$ 8.2. The experiment was performed using an electrophoresis mini set from BioRad (USA). The electrophoresis behaviors of the nanotubes were compared with standard natural DNA (i.e., GeneRuler DNA Ladder Mix, Thermo Fisher Scientific, Inc., Waltham, MA, USA). The gel was stained according to the manufacturer instruction (SYBR Gold nucleic-acid gel stain, from Molecular Probes Inc., Eugene, Oregon, USA).

Purification and Extraction of Magnetic DNA nanotubes from electrophoresis gel Extraction of DNA nanotubes from gel is required for purification of DNA origami products from unwanted nanostructures. For extraction of DNA nanotubes from the gel, "Quantum Prep Freeze N Squeeze DNA Gel-Extraction Spin Column, as a gel-specific kit from Bio-Rad (USA) was used". Initially, using a clean razor blade, carefully excise the bond of interest. Trim excess agarose from all six sides of the DNA nanotubes bond to maximize recovery and purity. Then the trimmed gel slice was chopped and placed the pieces in the filter cup of dolphin shaped microtube in $-20^{\circ} \mathrm{C}$ freezer for 5 minutes. The slice was then centrifuged in a gel extraction column with a proprietary tool and centrifuged at $13000 \mathrm{~g}$ for 3 minutes. The final product obtained from gel extraction, used as tubular DNA nanotubes incorporated of magnetic nanoparticles, was used for characterization studies.

\section{Microscopic characterization of DNA nanotubes}

Transmission electron microscope (TEM) was used to assess the size and morphology of magnetic DNA nanotubes and also the simple DNA nanotubes. For this purpose, the DNA nanotubes were immobilized by syringe spraying on Agar Scientific (Stansted, Essex CM24 8GF, United Kingdom) from carbon layer with 300-mesh $\mathrm{Cu}$ (50). The micrographs were obtained by TEM EM900 (Zeiss, Germany). Topographic characteristics of the nanotubes were studied by atomic force microscope (JPK-AFM, Germany). For this purpose, $5 \mu \mathrm{L}$ of the nanotubes was immobilized on a mica surface for 4 hours at $25{ }^{\circ} \mathrm{C}$. The AFM was performed in contact mode with JPK-AFM (with $150 \mathrm{~Hz}$ IGain, 0.0048 PGain, and 1.0 V set point via a JPK NanoWizard control). The cantilever was ACTA-10 probe model. The rough data were analyzed using the JPK Nano analyzer software.

\section{Results}

\section{Oligonucleotides as staples in DNA origami}

The computational design of the nanotube from cadnano software was indicated in Figure 2.

This platform showed a cylindrical scaffold assembled with the staples. The scaffold with 6 single-stranded DNA of M13mp18 nucleotide sequences that those were marked with blue color. The sequences of staples were determined as 24 oligonucleotides (Table 1) that those were marked with different colors in Figure 2.

\section{Microscopic characteristics}

TEM micrographs of DNA nanotubes without and included of magnetic nanoparticles were shown in Figure 3 and Figure 4.

The nanotubes were in filamentous shapes. The TEM 

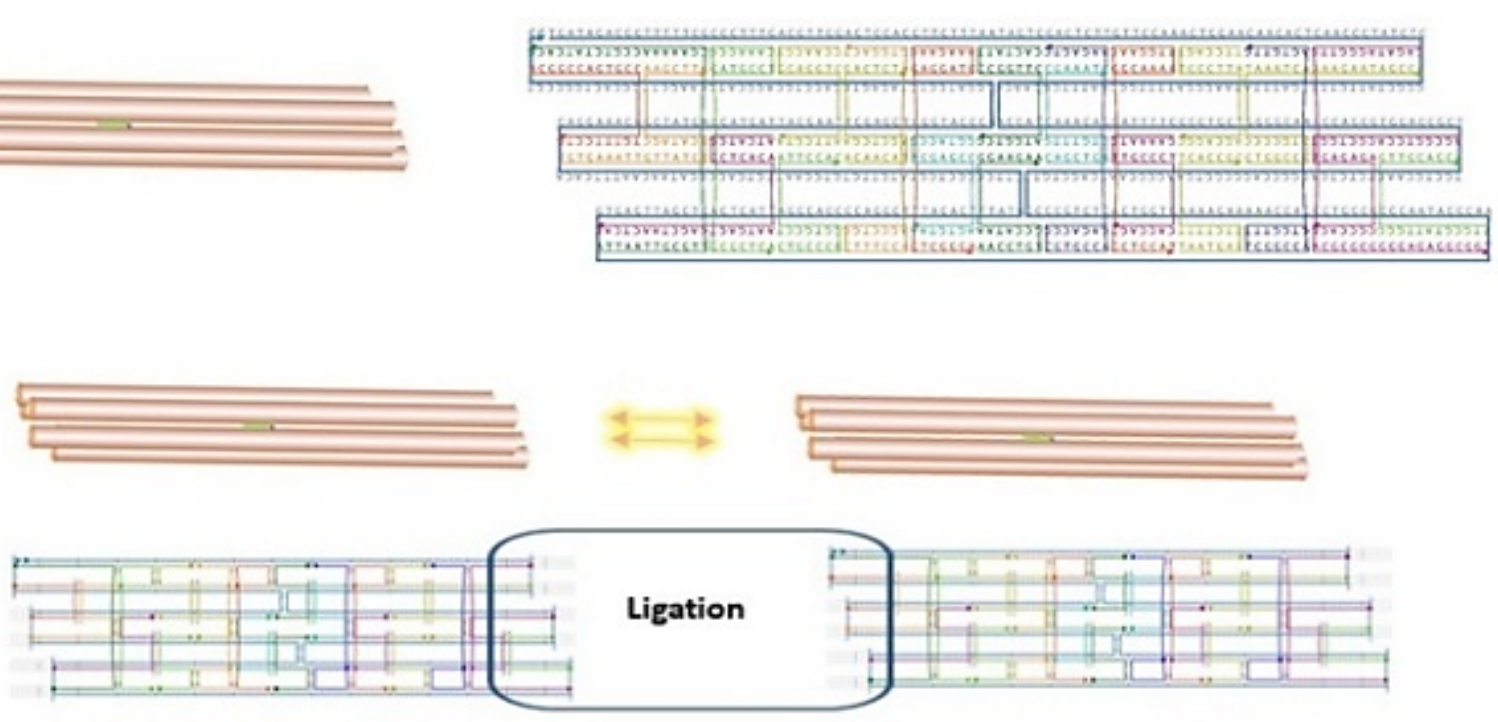

Figure 2: Schematic graphical view of DNA nanotubes designed in this study by cadnano.

Table 1: Oligonucleotide staples.

\begin{tabular}{|c|c|}
\hline No. & Sequence $\left(5^{\prime}\right.$ to $\left.3^{\prime}\right)$ \\
\hline 1 & CCAACGTGCAGGTCATTCGTA \\
\hline 2 & CACTATTCCGGTTCATGGTCG \\
\hline 3 & TTCCAGTTCCCTTAAGCAGGC \\
\hline 4 & GAGATAGGGTTGACGCGCGGGGAGAGGCGGT \\
\hline 5 & ACGGCCAGTGCCTGTTTCCTG \\
\hline 6 & CATGCCTCAAAGGGGCGCTCA \\
\hline 7 & GAGGATCAAAGAACGTCGGGA \\
\hline 8 & GGCAAAATTGGAACGCTGCAT \\
\hline 9 & ATCATGGGCTCACAAATGAGTGAGCTAACTCAC \\
\hline 10 & GGTACCGACGAGCCAGTGTAA \\
\hline 11 & GAAAATCTTGCCCTCACCAGT \\
\hline 12 & CATGCCTCAAAGGGGCGCTCA \\
\hline 13 & TGTGAAATTGTTATCCTCATAGCAAGCTTG \\
\hline 14 & ACAACATAGCTCGAGACTCTA \\
\hline 15 & CAGCTGACTGTTTGCGAAATC \\
\hline 16 & CTGGCCCTTGCCCCTAAATCAAAAGAATAGCCC \\
\hline 17 & AGCCTGGCTTTCCAGTGGACT \\
\hline 18 & GAGACGGCGTGCCAAAGAGTC \\
\hline 19 & GTGGTTTTCGGCCAAGTGTTG \\
\hline 20 & TTGCGTATTGGGGTTGCAGCA \\
\hline 21 & ATTAATTGCGTTCGAAAAACCGTCTATCACG \\
\hline 22 & CTGCCCGGGTGCCTATTCCAC \\
\hline 23 & AACCTGTGCCATAAGGAAGAA \\
\hline 24 & TAATGAATTCTTTTTCACCGC \\
\hline
\end{tabular}

micrograph was demonstrated encapsulation of C-MNPs inside the DNA nanotubes, obviously. The length of these nanotubes were estimated $\sim 1000 \mathrm{~nm}$ with $\sim 100$ $\mathrm{nm}$ diameter.

AFM results also demonstrated the nanoparticle-filled nanotubes (Figure 5); however, the free C-MNPs among DNA nanotubes were also seen that

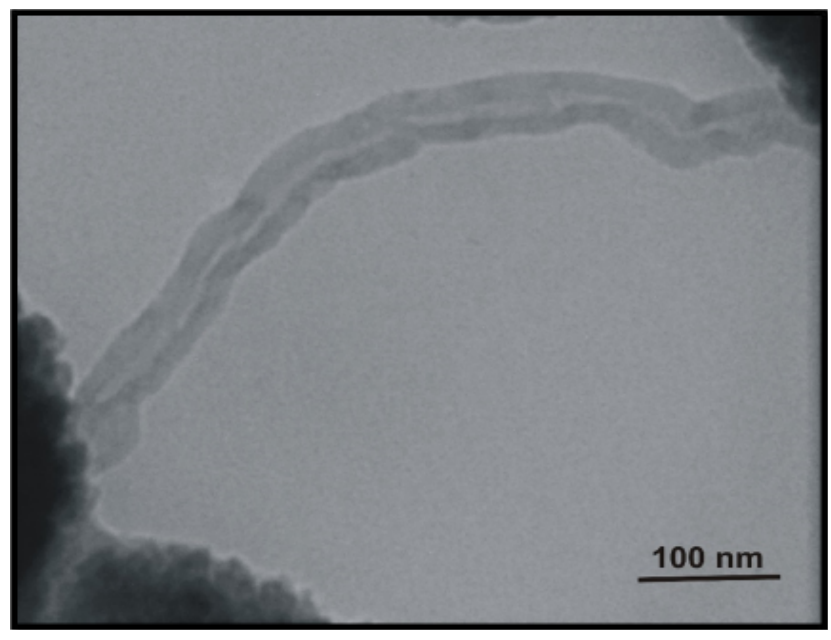

Figure 3: TEM micrograph of DNA nanotubes without of MNP.

those were not encapsulated within the nanotubes. Constructed-simultaneously encapsulation of magnetic nanoparticles into the large channel of this tubes leads to "pea-pod" particle alignment in nanotube channel which is visible in AFM topography view.

\section{Electrophoretic behaviors}

The electrophoretic behavior of DNA nanotubes contained C-MNPs was reversed in comparison to that behavior by natural DNA (e.g. DNA ladder) (Figure 6).

The nanoparticle-filled up nanotubes indicated positively charges and those moved toward the negative pole, whereas the natural DNA with the negatively charges moved to the anode. This behavior from the magnetic DNA nanotubes confirmed successfully the encapsulation of the cationic-charged MNPs by DNA nanotubes within their fabrication process.

\section{Discussion}

The principles of DNA nanotubes design and fabrication used in this research were reported pre- 

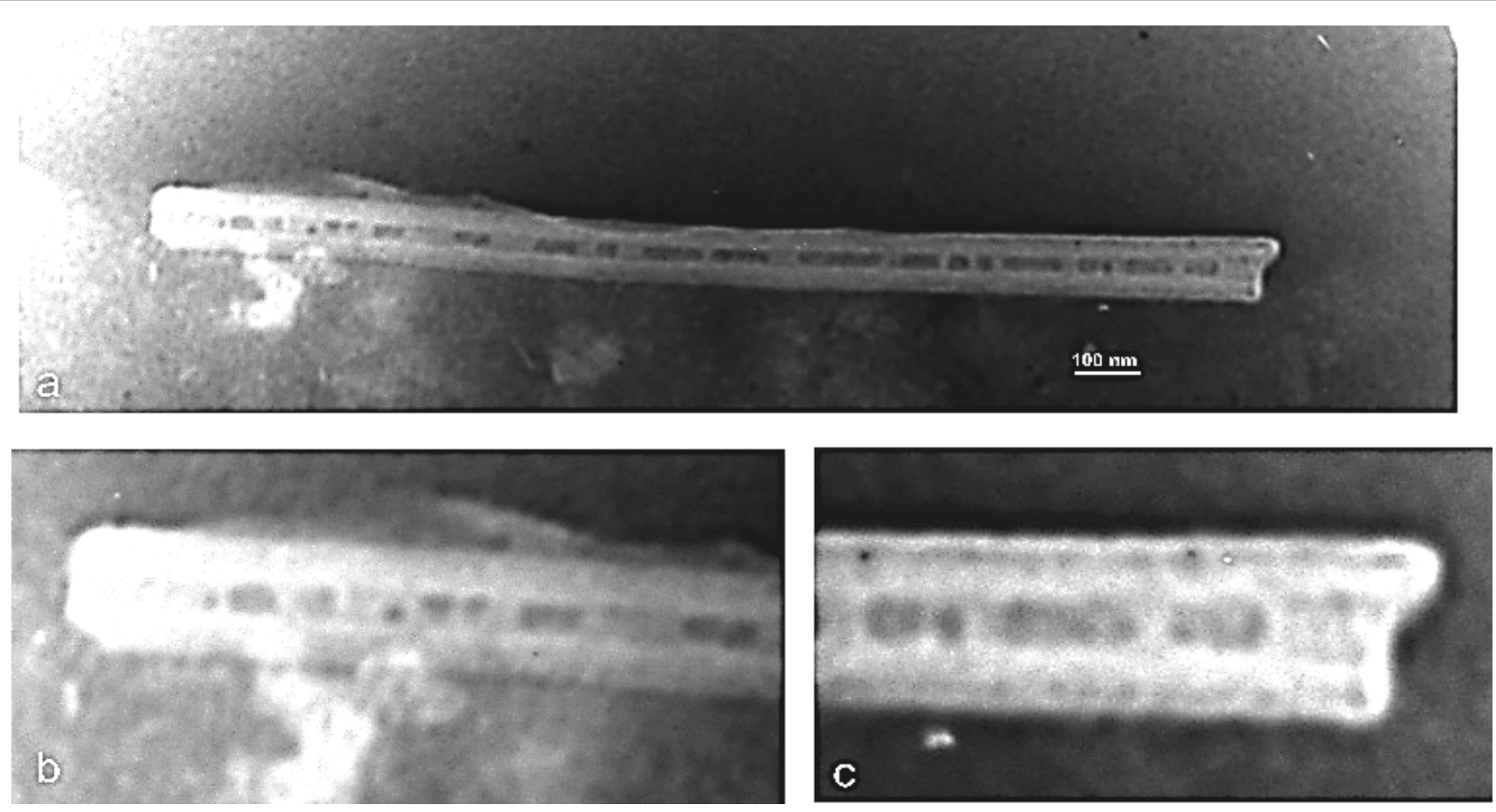

Figure 4: a) TEM micrograph of DNA nanotube contained MNPs; b,c) Maximized ends of the nanotube shown at 'a' image that those demonstrated MNPs densely in the channel of DNA nanotube.

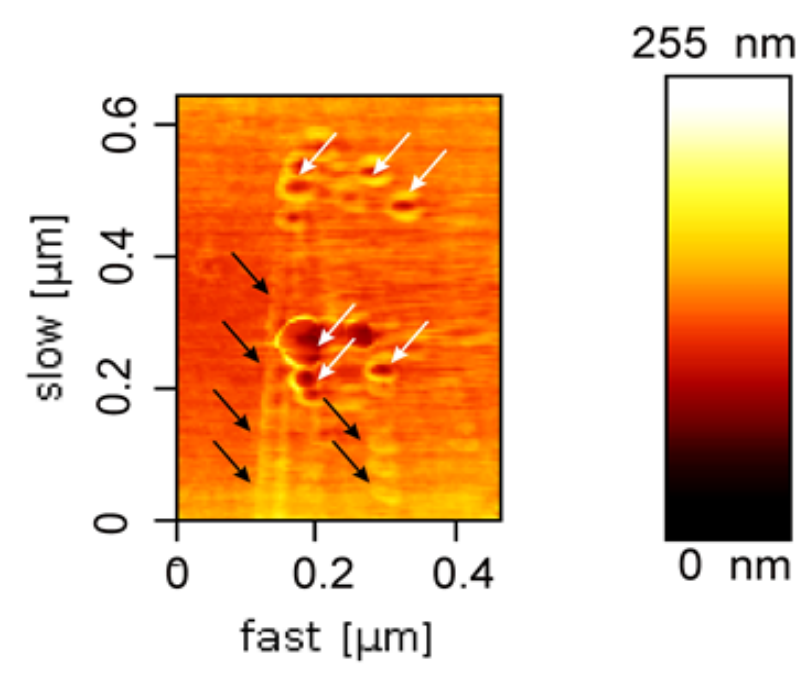

Figure 5: AFM of DNA nanotubes filled up with an array of C-MNPs (black arrows). Also, a few magnetic nanoparticles outside of DNA nanotubes were indicated (white arrows).

viously $[18,26,27]$. Here 24 staple sequences were determined for properly folding DNA nanotubes in the presences of cationic charged magnetic nanoparticles. The electrophoretic behavior of DNA nanotubes was previously studied. Simple DNA nanotubes showed the migration similarly to natural DNA from negative pole (cathode electrode) to positive pole (anode electrode) due to their negatively charges from the phosphate groups on their surfaces. While, the DNA nanotubes contained cationic charged magnetic nanoparticles fabricated in this study showed different electrophoresis behavior when compared with that by the natural DNA (Figure 5). Magnetic DNA nanotubes indicated positively charges due to the encapsulations of the cationic charged nanoparticles and their movements toward to the negatively pole, whereas the natural DNA and the empty DNA nanotubes have been showed migrations from the cathode electrode to the anode electrode within the electrophoresis experiment. The electrophoretic mobility of the magnetic DNA nanotubes suggested the encapsulations of the cationic-charged MNPs by DNA nanotubes; however, the AFM micrograph (Figure 4) demonstrated that the nanoparticles have being encapsulated inside the nanotubes, because in AFM image, it's obvious the periodic array or pea-pod alignment of magnetic nanoparticles in DNA nanotubes channel. In addition, it seemed that the addition of the nanoparticles in the origami reaction could potentially affect the fabrication of the magnetic nanotubes.

\section{Conclusion}

In conclusion, we have constructed nanoparticle-filled up DNA nanotubes via a DNA origami reaction contained cationic magnetic nanoparticles. DNA self-assembly was employed via the Origami reaction for obtaining well oriented DNA nanostructures with the affordable precision. The top-down approached fabrication would able to organize self-assembling the nanotubes with the hierarchically defined platforms. By incorporating the other DNA based molecules such as DNAzymes, aptamers, or DNA walkers, the nanotubes could be employed for a wide range of the applications in biomedicine [28-34]. For instance, these DNA nanotubes would be employed for the development of the high-throughput DNA biosensors. This 

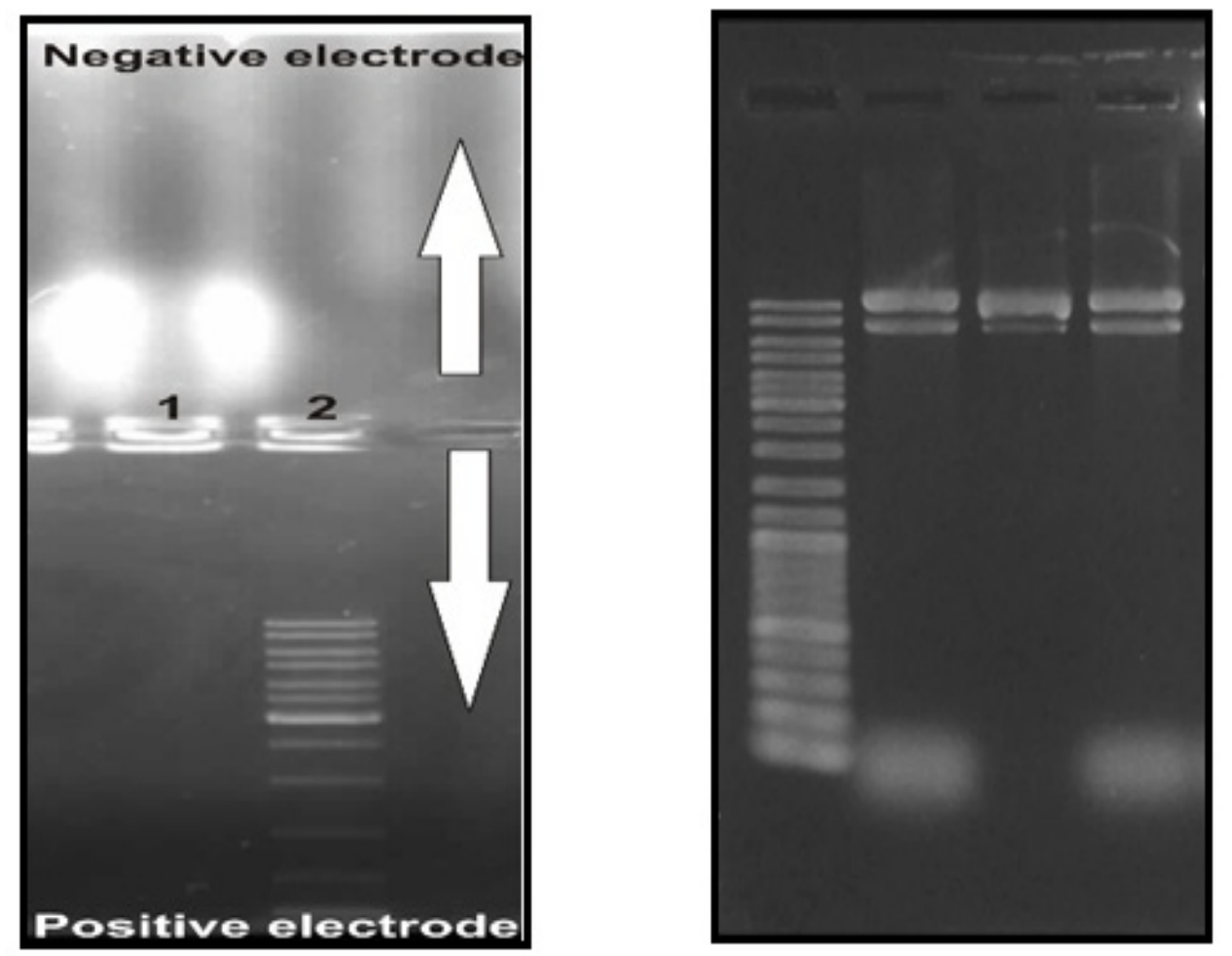

Figure 6: Electrophoretic movement of DNA nanotubes without and contained C-MNPs respectively (right and Left) in comparison to DNA ladder.

work presented herein using a procedure for filling-up DNA nanotubes with the cationic charged magnetic nanoparticles suggested as the hybrid nanomaterial for carrying biomolecules [35].

\section{Conflicts of Interest}

There are no conflicts to declare.

\section{Acknowledgements}

University of Isfahan (grant No. A/40284/94) and Mazandaran University of Medical Sciences (grant no. 1271/93) were supported this study. The authors thank also Central Research Laboratory, Mazandaran university of Medical Sciences and Iran Nanotechnology Initiative (INI).

\section{Author Contributions}

A.R. and P.G. conceived and designed the experiments; A.R. performed the experiments; P.G. and A.R analyzed the data; A.Z. and P.G. contributed reagents/ materials/analysis tools; A.R. and P.G. wrote the paper.

\section{References}

1. NC Seeman (2003) DNA in a material world. Nature 421: 427-431.

2. Tim Liedl, Thomas L Sobey, Friedrich C Simmel (2007) DNA-based nanodevices. Nanotoday 2: 36-41.

3. NC Seeman (1982) Nucleic acid junctions and lattices. J Theor Biol 99: 237-247.

4. ELF Winfree, LA Wenzler, NC Seeman (1998) Design and self-assembly of two-dimensional DNA crystals. Nature 394: 539-544.
5. S Sadhasivam, K S Yun (2010) DNA self-assembly: Prospectus and its future application. J Mater Sci 45: 25432552.

6. PWK Rothemund (2006) Folding DNA to create nanoscale shapes and patterns. Nature 440: 297-302.

7. C E Castro, F Kilchherr, D Kim, Shiao EL, Wauer T, et al. (2011) A primer to scaffolded DNA origami. Nature Method 8: 221-229.

8. F Hong, F Zhang, Z Y Liu, H Yan (2017) DNA origami: Scaffolds for creating higher order structures. Chem Rev 117: 12584-12640.

9. Samantaa, I L Medintz (2016) Nanoparticles and DNA - a powerful and growing functional combination in bionanotechnology. Nanoscale 8: 9037-9095.

10. S Julin, S Nummelin, MA Kostiainen, V Linko (2018) DNA nanostructure-directed assembly of metal nanoparticle superlattices. J Nanopart Res 20: 119.

11. J Sharma, R Chhabra, A Cheng, Jonathan Brownell, Yan Liu, et al. (2009) Control of self-assembly of DNA tubules through integration of gold nanoparticles. Science 323: 112-116.

12. H Li, S Ha Park, JH Reif, Thomas H LaBean, Hao Yan (2004) DNA-templated self-assembly of protein and nanoparticle linear arrays. J Am Chem Soc 126: 418-419.

13. Q Liu, C Song, Z G Wang, Na Li, Baoquan Ding (2014) Precise organization of metal nanoparticles on DNA origami template. Methods 67: 205-214.

14. B Teschome, S Facsko, K V Gothelf, Keller A (2015) Alignment of gold nanoparticle-decorated DNA origami nanotubes: Substrate prepatterning versus molecular combing. Langmuir 31: 12823-12829.

15. Y Liu, C Lin, H Li, Hao Yan (2005) Aptamer-directed self-assembly of protein arrays on a DNA nanostructure. Angewandte Chemie International Edition 44: 4333-4338. 
16. J Malo, C Vénien-Bryan, JR Harris, J Robin Harris, Holger Wille, et al. (2005) Engineering a 2D protein-DNA crystal. Angew Chem Int Ed Engl 44: 3057-3061.

17. Kuzyk, R Schreiber, Z Fan, Pardatscher, Eva-Maria Roller, et al. (2012) DNA-based self-assembly of chiral plasmonic nanostructures with tailored optical response. Nature 483: 311-314.

18. M Mousavi-Khatat, A Rafati, P Gill (2015) Fabrication of DNA nanotubes using origami-based nanostructures with sticky ends. J Nanostruct Chem 5: 177-183.

19. JD Le, Y Pinto, NC Seeman, K Musier-Forsyth, T Andrew Taton, et al. (2004) DNA-templated self-assembly of metallic nanocomponent arrays on a surface. Nano Letters 4: 2343-2347.

20. J Zhang, Y Liu, Y Ke, Yan H (2006) Periodic square-like gold nanoparticle arrays templated by self-assembled $2 \mathrm{D}$ DNA Nanogrids on a surface. Nano Letters 6: 248-251.

21. J Zheng, PE Constantinou, C Micheel, Alivisatos AP, Kiehl RA, et al. (2006) Two-dimensional nanoparticle arrays show the organizational power of robust DNA motifs. Nano Lett 6: 1502-1504.

22. BA Williams, K Lund, Y Liu, Yan H, Chaput JC (2007) Self-assembled peptide nanoarrays: An approach to studying protein-protein interactions. Angew Chem Int Ed Engl 46: 3051-3054.

23. Y He, Y Tian, AE Ribbe, C Mao (2006) Antibody nanoarrays with a pitch of -20 nanometers. J Am Chem Soc 128: 12664-12665.

24. C Lin, Y Ke, Y Liu, Michael Mertig, Jian Gu, et al. (2007) Functional DNA nanotube arrays: Bottom-up meets topdown. Angew Chem Int Ed Engl 46: 6089-6092.

25. V Pavlov, Y Xiao, R Gill, Dishon A, Kotler M, et al. (2004) Amplified chemiluminescence surface detection of DNA and telomerase activity using catalytic nucleic acid labels.
Anal Chem 76: 2152-2156.

26. Rafati, P Gill (2015) Ultrastructural characterizations of DNA nanotubes using scanning tunneling and atomic force microscopes. Journal of Microscopy and Ultrastructure 4: $1-5$.

27. H Bui, C Onodera, C Kidwell, Tan Y, Graugnard E, et al. (2010) Programmable periodicity of quantum dot arrays with DNA origami nanotubes. Nano Lett 10: 3367-3372.

28. MN Stojanovic, DA Stefanovic (2003) A deoxyribozyme-based molecular automaton. Nature Biotechnology 21: 1069-1074.

29. R Nutiu, S Mei, Z Liu, Yingfu Li (2004) Engineering DNA aptamers and DNA enzymes with fluorescence-signaling properties. Pure Appl Chem 76: 1547-1561.

30. JS Shin, NA Pierce (2005) A synthetic DNA walker for molecular transport. J Am Chem Soc 126: 10834-10835.

31. Y Tian, Y He, Y Chen, Yin P, Mao C (2005) A DNAzyme that walks processively and autonomously along a one-dimensional track. Angew Chem Int Ed Engl 44: 4355-4358.

32. R Pei, SK Taylor, D Stefanovic, Rudchenko S, Mitchell TE, et al. (2006) Behavior of polycatalytic assemblies in a substrate-displaying matrix. J Am Chem Soc 128: 1269312699.

33. AM Mohammed, P Sulc, J Zenk, Rebecca Schulman (2017) Self-assembling DNA nanotubes to connect molecular landmarks. Nature Nanotechnology 12: 312-316.

34. AM Mohammed, L Velazquez, A Chisenhall, D Schiffels, DK Fygenson, et al. (2017) Self-assembly of precisely defined DNA nanotube superstructures using DNA origami seeds. Nanoscale 9: 522-526.

35. Rafati, A Zarrabi, P Gill (2017) Fabrication of DNA nanotubes with an array of exterior magnetic nanoparticles. Mater Sci Eng C Mater Biol Appl 79: 216-220. 